\title{
An Impulsive Periodic Single-Species Logistic System with Diffusion
}

\author{
Chenxue Yang, ${ }^{1,2}$ Mao Ye, ${ }^{1,2}$ and Zijian Liu ${ }^{3,4}$ \\ ${ }^{1}$ School of Computer Science and Engineering, University of Electronic Science and Technology of China, Chengdu 611731, China \\ ${ }^{2}$ State Key Laboratory for Novel Software Technology, Nanjing University, Nanjing 210093, China \\ ${ }^{3}$ School of Science, Chongqing Jiaotong University, Chongqing 400074, China \\ ${ }^{4}$ Department of Mathematics, Hangzhou Normal University, Hangzhou, Zhejiang 310036, China
}

Correspondence should be addressed to Zijian Liu; hbliuzijian@126.com

Received 11 January 2013; Accepted 17 April 2013

Academic Editor: Michael Meylan

Copyright (C) 2013 Chenxue Yang et al. This is an open access article distributed under the Creative Commons Attribution License, which permits unrestricted use, distribution, and reproduction in any medium, provided the original work is properly cited.

\begin{abstract}
We study a single-species periodic logistic type dispersal system in a patchy environment with impulses. On the basis of inequality estimation technique, sufficient conditions of integrable form for the permanence and extinction of the system are obtained. By constructing an appropriate Lyapunov function, conditions for the existence of a unique globally attractively positive periodic solution are also established. Numerical examples are shown to verify the validity of our results and to further discuss the model.
\end{abstract}

\section{Introduction}

In the practical world, on the one hand, owing to natural enemy, severe competition, or deterioration of the patch environment, species dispersal in two or more patches becomes one of the most prevalent phenomena of nature. Many empirical works and monographies on population dynamics in a spatial heterogeneous environment have been done (see [1-9] and the references cited therein). On the other hand, many natural and man-made factors (e.g., fire, drought, flooding deforestation, hunting, harvesting, breeding, etc.) always lead to rapid decrease or increase of population number at fixed moment. Such sudden changes can often be characterized mathematically in the form of impulses. With the development of the theory of impulsive differential equations [10], various population dynamical models of impulsive differential equations have been proposed and studied extensively. For example, many important and interesting results on the permanence, persistence, extinction, global stability, the existence of positive periodic solutions, bifurcation and dynamical complexity, and so forth can be found in [11-17] and the references cited therein.

Although considerable researches on the dispersal and impulses of species have been reported in the literature, there are few papers that investigate the dynamical behavior of population systems under the circumstances in which both dispersal and impulse exist. However, dispersal species which undergoes impulses is also one of the most prevalent phenomena of nature. In our previous paper [18], an impulsive periodic predator-prey system with diffusion is studied, and some conditions for the permanence, extinction, and existence of a unique globally stable periodic solution are established. In this paper, we will present and study a singlespecies periodic logistic system with impulses and dispersal in $n$ different patches. Our model takes the form

$$
\begin{aligned}
\dot{x}_{i}(t)= & x_{i}(t)\left(r_{i}(t)-a_{i}(t) x_{i}(t)\right) \\
& +\sum_{j=1}^{n} D_{i j}(t)\left(x_{j}(t)-x_{i}(t)\right), \quad t \neq t_{k}, \\
x_{i}\left(t_{k}^{+}\right)= & h_{i k} x_{i}\left(t_{k}\right), \quad i=1,2, \ldots, n, k=1,2, \ldots,
\end{aligned}
$$

where $r_{i}(t)$ and $a_{i}(t)(i \in I=\{1,2, \ldots, n\})$ represent the intrinsic growth rates and the density-dependent coefficients in patch $i$, respectively. $D_{i j}(t) \geqslant 0(i, j \in I)$ denotes the dispersal rate of the species from patch $j$ to patch $i$. $h_{i k} x_{i}\left(t_{k}\right)(i \in I)$ is the regular pulse at time $t_{k}$ of species 
$x$ in patch $i$. Throughout this paper, we always assume the following.

$\left(C_{1}\right) r_{i}(t), a_{i}(t)$, and $D_{i j}(t)(i, j \in I)$ are continuously periodic functions with common period $T$, defined on $R_{+}=[0, \infty)$ and $a_{i}(t) \geqslant 0, D_{2} \geqslant D_{i j}(t) \geqslant D_{1}>$ $0(i \neq j), D_{i i}(t) \equiv 0$ for all $i, j \in I$ and $t \in R_{+}$.

$\left(C_{2}\right) h_{i k}>0$ for all $i \in I, k=1,2, \ldots$ and there exists a positive integer $q$ such that $t_{k+q}=t_{k}+T$ and $h_{i(k+q)}=$ $h_{i k}$ for any $i \in I$.

The organization of this paper is as follows. In the next section, some sufficient conditions for the permanence and extinction of system (1) are obtained. In Section 3, conditions for the existence of a unique globally attractively positive periodic solution are also established. Finally, some numerical simulations are proposed to illustrate the feasibility of our results and discuss the model further.

\section{Permanence and Extinction}

In this section, applying inequality estimation technique, we get some sufficient conditions on the permanence and extinction of system (1).

Theorem 1. There exists a positive constant $M$ such that $\lim \sup _{t \rightarrow \infty} x_{i}(t)<M$ for all $i \in I$ if

$$
\int_{0}^{T} a(t) \mathrm{d} t>0
$$

where $a(t)=\min _{i \in I}\left\{a_{i}(t)\right\}$.

Proof. Let $h_{k}=\max _{i \in I}\left\{h_{i k}\right\}$ for any $k=1,2, \ldots$, then we have $h_{k+q}=h_{k}$ and there exists a positive constant $H$ such that function

$$
|h(t, \mu)|=\left|\sum_{t \leqslant t_{k}<t+\mu} \ln h_{k}\right| \leqslant \sum_{k=1}^{q}\left|\ln h_{k}\right| \leqslant H
$$

for all $t \in R_{+}$and $\mu \in[0, T]$. Choose $r(t)=\max _{i \in I}\left\{r_{i}(t)\right\}$, and $r(t)$ is bounded for all $t \in R_{+}$. Then from conditions (2) and (3), we have two positive constants $\tau$ and $\delta$ such that

$$
\int_{0}^{T}(r(t)-a(t) \tau) \mathrm{d} t+\sum_{k=1}^{q} \ln h_{k}<-\delta .
$$

Define the function $V(t)=\max _{i \in I}\left\{x_{i}(t)\right\}$. For any $t \in R_{+}$, there is an $i=i(t) \in I$ such that $V(t)=x_{i}(t)$. Calculating the upper-right derivative of $V(t)$, we obtain

$$
\begin{aligned}
D^{+} V(t) & \leqslant x_{i}(t)\left(r_{i}(t)-a_{i}(t) x_{i}(t)\right) \\
& \leqslant V(t)(r(t)-a(t) V(t)) .
\end{aligned}
$$

When $t=t_{k}$, we have $V\left(t_{k}^{+}\right)=\max \left\{x_{i}\left(t_{k}^{+}\right)\right\}=$ $\max \left\{h_{i k} x_{i}\left(t_{k}\right)\right\} \leqslant \max \left\{h_{i k}\right\} \max \left\{x_{i}\left(t_{k}\right)\right\}=h_{k} V\left(t_{k}\right)$.

Consider the following auxiliary system:

$$
\begin{gathered}
D^{+} w(t)=w(t)(r(t)-a(t) w(t)), \\
w\left(t_{k}^{+}\right)=h_{k} w\left(t_{k}\right), \quad k=1,2, \ldots
\end{gathered}
$$

with the initial condition $V(0) \leqslant w(0)$. If there is a constant $M_{0}>0$ such that

$$
\limsup _{t \rightarrow \infty} w(t)<M_{0}
$$

for any positive solution $w(t)$ of system (6), then, according to the comparison theorem of impulsive differential equations [10], we have $V(t) \leqslant w(t)$ for all $t \geqslant 0$. Therefore, choose $M=M_{0}$, and we will finally have $\limsup _{t \rightarrow \infty} x_{i}(t) \leqslant$ $\limsup _{t \rightarrow \infty} V(t) \leqslant \limsup _{t \rightarrow \infty} w(t)<M$ for all $i \in I$.

Next, we will prove that $(7)$ holds. In fact, for any positive solution $w(t)$ of system (6), we only need to consider the following three cases.

Case 1. There is a $t_{0} \geqslant 0$ such that $w(t) \geqslant \tau$ for all $t \geqslant t_{0}$.

Case 2. There is a $t_{0} \geqslant 0$ such that $w(t) \leqslant \tau$ for all $t \geqslant t_{0}$.

Case 3. $w(t)$ is oscillatory about $\tau$ for all $t \geqslant 0$.

We first consider Case 1. Since $w(t) \geqslant \tau$ for all $t \geqslant t_{0}$, then for $t=t_{0}+l T$, where $l$ is any positive integer, integrating system (6) from $t_{0}$ to $t$, from (4) we have

$$
\begin{gathered}
w(t)=w\left(t_{0}\right) \exp \left(\int_{t_{0}}^{t}(r(s)-a(s) w(s)) \mathrm{d} s+\sum_{t_{0} \leqslant t_{k}<t} \ln h_{k}\right) \\
\leqslant w\left(t_{0}\right) \exp \left(\int_{t_{0}}^{t_{0}+T}(r(s)-a(s) \tau) \mathrm{d} s+\cdots\right. \\
+\int_{t_{0}+(l-1) T}^{t_{0}+l T}(r(s)-a(s) \tau) \mathrm{d} s \\
\left.+l \sum_{k=1}^{q} \ln h_{k}\right) \\
\leqslant w\left(t_{0}\right) \exp (-l \delta) .
\end{gathered}
$$

Hence, $w(t) \rightarrow 0$ as $l \rightarrow \infty$, which leads to a contradiction.

Then, we consider Case 3. From the oscillation of $w(t)$ about $\tau$, we can choose two sequences $\left\{\rho_{n}\right\}$ and $\left\{\rho_{n}^{*}\right\}$ satisfying $0<\rho_{1}<\rho_{1}^{*}<\cdots<\rho_{n}<\rho_{n}^{*}<\cdots$ and $\lim _{n \rightarrow \infty} \rho_{n}=$ $\lim _{n \rightarrow \infty} \rho_{n}^{*}=\infty$ such that

$$
\begin{aligned}
& w\left(\rho_{n}\right) \leqslant \tau, \quad w\left(\rho_{n}^{+}\right) \geqslant \tau, \\
& w\left(\rho_{n}^{*}\right) \geqslant \tau, \quad w\left(\rho_{n}^{*+}\right) \leqslant \tau, \\
& w(t) \geqslant \tau \quad \forall t \in\left(\rho_{n}, \rho_{n}^{*}\right), \\
& w(t) \leqslant \tau \quad \forall t \in\left(\rho_{n}^{*}, \rho_{n+1}\right) .
\end{aligned}
$$

For any $t \geqslant \rho_{1}$, if $t \in\left(\rho_{n}, \rho_{n}^{*}\right]$ for some integer $n$, then we can choose integer $l \geqslant 0$ and constant $0 \leqslant v<T$ such that $t=\rho_{n}+l T+\nu$. Since $D^{+} w(t) \leqslant w(t)(r(t)-a(t) \tau)$ for all 
$t \in\left(\rho_{n}, \rho_{n}^{*}\right)$ and $t \neq t_{k}$, integrating this inequality from $\rho_{n}$ to $t$, by (3) and (4) we obtain

$$
\begin{gathered}
w(t) \leqslant w\left(\rho_{n}\right) \exp \left(\int_{\rho_{n}}^{t}(r(s)-a(s) \tau) \mathrm{d} s+\sum_{\rho_{n} \leqslant t_{k}<t} \ln h_{k}\right) \\
\leqslant \tau \exp \left(l\left(\int_{0}^{T}(r(t)-a(t) \tau) \mathrm{d} t+\sum_{k=1}^{q} \ln h_{k}\right)\right. \\
\left.+\int_{\rho_{n}}^{\rho_{n}+v}(r(t)-a(t) \tau) \mathrm{d} t+\sum_{\rho_{n} \leqslant t_{k}<\rho_{n}+\nu} \ln h_{k}\right) \\
\leqslant \tau \exp (-l \delta+r T+H) \leqslant \tau \exp (r T+H),
\end{gathered}
$$

where $r=\sup _{t \in[0, T]}\{|r(t)|+a(t) \tau\}$. If there is an integer $n$ such that $t \in\left(\rho_{n}^{*}, \rho_{n+1}\right]$, then we have $w(t) \leqslant \tau \leqslant \tau \exp (r T+H)$. Therefore, for Case 3 we always have $w(t) \leqslant \tau \exp (r T+H)$ for all $t \geqslant \rho_{1}$.

Lastly, if Case 2 holds, then we directly have $w(t) \leqslant$ $\tau \exp (r T+H)$ for all $t \geqslant 0$.

Choose constant $M_{0}=\tau \exp (r T+H)+1$, then we see that (7) holds. This completes the proof.

Remark 2. It can be seen from Theorem 1 that, in one time period $T$, if the density-dependent coefficient in patch $i$ ( $i \in$ $I)$ is strictly greater than zero and the impulsive coefficient $h_{i k}$ is bounded in the same time period, the dispersal species $x$ is always ultimately bounded.

Theorem 3. Assume that all conditions of Theorem 1 hold. In addition, there is an $i_{0} \in I$ such that

$$
\int_{0}^{T}\left(r_{i_{0}}(t)-\sum_{j=1}^{n} D_{i_{0} j}(t)\right) \mathrm{d} t+\sum_{k=1}^{q} \ln h_{i_{0} k}>0 .
$$

Then system (1) is permanent.

Proof. The ultimate boundedness of system (1) has been proved in Theorem 1 . In the following, we mainly prove the permanence of the system; that is, there is a constant $m>0$ such that

$$
\liminf _{t \rightarrow \infty} x_{i}(t)>m
$$

for each $i \in I$ and any positive solution $x(t)=\left(x_{1}(t), x_{2}(t)\right.$, $\left.\ldots, x_{n}(t)\right)$ of system (1).

From assumption $h_{i(k+q)}=h_{i k}$, we obtain that there exists a constant $H>0$ such that function

$$
\left|h_{i}(t, \mu)\right|=\left|\sum_{t \leqslant t_{k}<t+\mu} \ln h_{i k}\right| \leqslant \sum_{k=1}^{q}\left|\ln h_{i k}\right| \leqslant H
$$

for any $i \in I, t \in R_{+}$and $\mu \in[0, T]$.

For $i=i_{0}$, by condition (11) and the boundedness of $a_{i_{0}}(t)$, there are two positive constants $\bar{\tau}$ and $\bar{\delta}$ such that

$$
\int_{0}^{T}\left(r_{i_{0}}(t)-\sum_{j=1}^{n} D_{i_{0} j}(t)-a_{i_{0}}(t) \bar{\tau}\right) \mathrm{d} t+\sum_{k=1}^{q} \ln h_{i_{0} k}>\bar{\delta} .
$$

Let $x(t)=\left(x_{1}(t), x_{2}(t), \ldots, x_{n}(t)\right)$ be any positive solution of system (1). Since

$$
\begin{array}{r}
\dot{x}_{i_{0}}(t) \geqslant x_{i_{0}}(t)\left(r_{i_{0}}(t)-\sum_{j=1}^{n} D_{i_{0} j}(t)-a_{i_{0}}(t) x_{i_{0}}(t)\right), \\
t \neq t_{k},
\end{array}
$$

by the comparison theorem of impulsive differential equations, we obtain $x_{i_{0}}(t) \geqslant u(t)$ for all $t \geqslant 0$, where $u(t)$ is the positive solution of system

$$
\begin{array}{r}
\dot{u}(t)=u(t)\left(r_{i_{0}}(t)-\sum_{j=1}^{n} D_{i_{0} j}(t)-a_{i_{0}}(t) u(t)\right), \\
u\left(t_{k}^{+}\right)=h_{i_{0} k} u\left(t_{k}\right), \quad k=1,2, \ldots
\end{array}
$$

with initial condition $u(0)=x_{i_{0}}(0)$.

In the following, we first prove that there is a constant $\bar{m}>$ 0 such that

$$
\liminf _{t \rightarrow \infty} u(t)>\bar{m}
$$

for any positive solution $u(t)$ of system (16). We only need to consider the following three cases.

Case 1 . There is a $\bar{t} \geqslant 0$ such that $u(t) \leqslant \bar{\tau}$ for all $t \geqslant \bar{t}$.

Case 2. There is a $\bar{t} \geqslant 0$ such that $u(t) \geqslant \bar{\tau}$ for all $t \geqslant \bar{t}$.

Case 3. $u(t)$ is oscillatory about $\bar{\tau}$ for all $t \geqslant 0$.

For Case 1 , let $t=\bar{t}+l T$, where $l \geqslant 0$ is any integer. From (14), we obtain

$$
\begin{aligned}
& u(t) \\
& =u(\bar{t}) \\
& \times \exp \left(\int_{\bar{t}}^{t}\left(r_{i_{0}}(s)-\sum_{j=1}^{n} D_{i_{0} j}(s)-a_{i_{0}}(s) u(s)\right) \mathrm{d} s\right. \\
& \left.+\sum_{\bar{t} \leqslant t_{k}<t} \ln h_{i_{0} k}\right) \\
& \geqslant u(\bar{t}) \exp \left(\int_{\bar{t}}^{\bar{t}+T}\left(r_{i_{0}}(s)-\sum_{j=1}^{n} D_{i_{0} j}(s)-a_{i_{0}}(s) \bar{\tau}\right) \mathrm{d} s\right. \\
& +\cdots+\int_{\bar{t}+(l-1) T}^{\bar{t} l T}\left(r_{i_{0}}(s)-\sum_{j=1}^{n} D_{i_{0} j}(s)\right. \\
& \left.-a_{i_{0}}(s) \bar{\tau}\right) \mathrm{d} s \\
& \left.+l \sum_{k=1}^{q} \ln h_{i_{0} k}\right) \\
& \geqslant u(\bar{t}) \exp (\bar{l} \bar{\delta}) .
\end{aligned}
$$


Hence, $u(t) \rightarrow \infty$ as $l \rightarrow \infty$, which leads to a contradiction.

For Case 3, we choose two sequences $\left\{\rho_{n}\right\}$ and $\left\{\rho_{n}^{*}\right\}$ satisfying $0<\rho_{1}<\rho_{1}^{*}<\cdots<\rho_{n}<\rho_{n}^{*}<\cdots$ and $\lim _{n \rightarrow \infty} \rho_{n}=\lim _{n \rightarrow \infty} \rho_{n}^{*}=\infty$ such that

$$
\begin{array}{cl}
u\left(\rho_{n}\right) \geqslant \bar{\tau}, \quad & u\left(\rho_{n}^{+}\right) \leqslant \bar{\tau}, \quad u\left(\rho_{n}^{*}\right) \leqslant \bar{\tau}, \quad u\left(\rho_{n}^{*+}\right) \geqslant \bar{\tau} \\
& u(t) \leqslant \bar{\tau} \quad \forall t \in\left(\rho_{n}, \rho_{n}^{*}\right), \\
& u(t) \geqslant \bar{\tau} \quad \forall t \in\left(\rho_{n}^{*}, \rho_{n+1}\right) .
\end{array}
$$

For any $t \geqslant \rho_{1}$, if $t \in\left(\rho_{n}, \rho_{n}^{*}\right]$ for some integer $n$, then we can choose integer $l \geqslant 0$ and constant $0 \leqslant \bar{v}<T$ such that $t=$ $\rho_{n}+l T+\bar{\nu}$. Since, for all $t \in\left(\rho_{n}, \rho_{n}^{*}\right)$ and $t \neq t_{k}$, we have $\dot{u}(t) \geqslant$ $u(t)\left(r_{i_{0}}(t)-\sum_{j=1}^{n} D_{i_{0} j}(t)-a_{i_{0}}(t) \bar{\tau}\right)$, integrating this inequality from $\rho_{n}$ to $t$, then from (13) and (14) we obtain

$$
\begin{aligned}
& u(t) \\
& \geqslant u\left(\rho_{n}\right) \\
& \times \exp \left(\int_{\rho_{n}}^{t}\left(r_{i_{0}}(s)-\sum_{j=1}^{n} D_{i_{0} j}(s)-a_{i_{0}}(s) \bar{\tau}\right) \mathrm{d} s\right. \\
& \left.+\sum_{\rho_{n} \leqslant t_{k}<t} \ln h_{i_{0} k}\right) \\
& \geqslant \bar{\tau} \exp \left(l \left(\int_{0}^{T}\left(r_{i_{0}}(t)-\sum_{j=1}^{n} D_{i_{0} j}(t)-a_{i_{0}}(t) \bar{\tau}\right) \mathrm{d} t\right.\right. \\
& \left.+\sum_{k=1}^{q} \ln h_{i_{0} k}\right) \\
& +\int_{\rho_{n}}^{\rho_{n}+\bar{\nu}}\left(r_{i_{0}}(t)-\sum_{j=1}^{n} D_{i_{0} j}(t)-a_{i_{0}}(t) \bar{\tau}\right) \mathrm{d} t \\
& \left.+\sum_{\rho_{n} \leqslant t_{k}<\rho_{n}+\bar{\nu}} \ln h_{i_{0} k}\right) \\
& \geqslant \bar{\tau} \exp \left(l \bar{\delta}-\beta_{i_{0}} T-H\right) \geqslant \bar{\tau} \exp \left(-\beta_{i_{0}} T-H\right),
\end{aligned}
$$

where $\beta_{i_{0}}=\sup _{t \in[0, T]}\left\{\left|r_{i_{0}}(t)\right|+\sum_{j=1}^{n} D_{i_{0} j}(t)+a_{i_{0}}(t) \bar{\tau}\right\}$. If there is an integer $n$ such that $t \in\left(\rho_{n}^{*}, \rho_{n+1}\right]$, obviously we have $u(t) \geqslant$ $\bar{\tau} \geqslant \bar{\tau} \exp \left(-\beta_{i_{0}} T-H\right)$. Therefore, for Case 3 we always have $u(t) \geqslant \bar{\tau} \exp \left(-\beta_{i_{0}} T-H\right)$ for all $t \geqslant \rho_{1}$. Let constant $\bar{m}=$ $\bar{\tau} \exp \left(-\beta_{i_{0}} T-H-1\right)$. Then $\bar{m}$ is independent of any positive solution of system (16) and we finally have that (17) holds.

Lastly, if Case 2 holds, then from $u(t) \geqslant \bar{\tau}$ for all $t \geqslant 0$, we directly have that (17) holds.

From the fact that $x_{i_{0}}(t) \geqslant u(t)$ for all $t \geqslant 0$, then we have

$$
\liminf _{t \rightarrow \infty} x_{i_{0}}(t) \geqslant \liminf _{t \rightarrow \infty} u(t)>\bar{m} .
$$

It follows immediately from (21) that there is a $\bar{T}_{0}>0$ such that $\sum_{i=1}^{n} x_{i}(t) \geqslant \bar{m}$ for all $t \geqslant \bar{T}_{0}$. Then for any $i \in I$, when $t \geqslant \bar{T}_{0}$ and $t \neq t_{k}$ we have

$$
\begin{aligned}
\dot{x}_{i}(t)= & x_{i}(t)\left(r_{i}(t)-a_{i}(t) x_{i}(t)-\sum_{j=1}^{n} D_{i j}(t)\right) \\
& +\sum_{j=1}^{n} D_{i j}(t) x_{j}(t) \\
\geqslant & x_{i}(t)\left(r_{i}(t)-a_{i}(t) x_{i}(t)-\sum_{j=1}^{n} D_{i j}(t)-D_{1}\right)+D_{1} \bar{m} .
\end{aligned}
$$

Obviously, there is a constant $\beta>0$ and $\beta$ is independent of any positive solution of system (1), such that for any $i \in I$, $0 \leqslant x_{i}(t) \leqslant \beta$, and all $t \in R_{+}$the following inequality holds

$$
x_{i}(t)\left(r_{i}(t)-a_{i}(t) x_{i}(t)-\sum_{j=1}^{n} D_{i j}(t)-D_{1}\right)+D_{1} \bar{m}>\beta
$$

Hence, for any $i \in I$, by (22) we have

$$
\begin{gathered}
\dot{x}_{i}(t)>\beta, \quad t \neq t_{k}, \\
x_{i}\left(t_{k}^{+}\right) \geqslant \tilde{h}_{i} x_{i}\left(t_{k}\right), \quad k=1,2, \ldots
\end{gathered}
$$

for all $0 \leqslant x_{i}(t) \leqslant \beta$ and $t \geqslant \bar{T}_{0}$, where $\widetilde{h}_{i}=\min _{1 \leqslant k \leqslant q}\left\{h_{i k}\right\}>0$. In order to prove that (12) holds, we only need to consider the following three cases.

Case 1. There is a $\bar{T}_{1} \geqslant \bar{T}_{0}$ such that $x_{i}(t) \geqslant \beta$ for all $t \geqslant \bar{T}_{1}$. Case 2. There is a $\bar{T}_{1} \geqslant \bar{T}_{0}$ such that $x_{i}(t) \leqslant \beta$ for all $t \geqslant \bar{T}_{1}$. Case 3. $x_{i}(t)$ is oscillatory about $\beta$ for all $t \geqslant \bar{T}_{0}$.

Equation (12) is obviously true if Case 1 holds.

For Case 2, there exists an impulsive time $t_{q^{*}} \geqslant \bar{T}_{1}$ for some integer $q^{*}>0$. For any $t>t_{q^{*}+1}>t_{q^{*}} \geqslant \bar{T}_{1}$, there is an integer $p \geqslant q^{*}+1$ such that $t \in\left(t_{p}, t_{p+1}\right]$, and from system (24) we have

$$
\begin{aligned}
x_{i}(t) & >x_{i}\left(t_{p}^{+}\right)+\beta\left(t-t_{p}\right) \geqslant \widetilde{h}_{i} x_{i}\left(t_{p}\right)+\beta\left(t-t_{p}\right) \\
& \geqslant \widetilde{h}_{i}\left[x_{i}\left(t_{p-1}^{+}\right)+\beta\left(t_{p}-t_{p-1}\right)\right]+\beta\left(t-t_{p}\right)>\widetilde{h}_{i} \beta \tau_{0},
\end{aligned}
$$

where $\tau_{0}=\min _{1 \leqslant k \leqslant q}\left\{t_{k}-t_{k-1}\right\}>0$. Therefore, we obtain

$$
\liminf _{t \rightarrow \infty} x_{i}(t)>\widetilde{h}_{i} \beta \tau_{0}
$$

for any $i \in I$. 
Then, we consider Case 3. We choose two sequences $\left\{\rho_{n}\right\}$ and $\left\{\rho_{n}^{*}\right\}$ satisfying $\bar{T}_{0}<\rho_{1}<\rho_{1}^{*}<\cdots<\rho_{n}<\rho_{n}^{*}<\cdots$ and $\lim _{n \rightarrow \infty} \rho_{n}=\lim _{n \rightarrow \infty} \rho_{n}^{*}=\infty$ such that

$$
\begin{aligned}
& x_{i}\left(\rho_{n}\right) \geqslant \beta, \quad x_{i}\left(\rho_{n}^{+}\right) \leqslant \beta, \\
& x_{i}\left(\rho_{n}^{*}\right) \leqslant \beta, \quad x_{i}\left(\rho_{n}^{*+}\right) \geqslant \beta, \\
& x_{i}(t) \leqslant \beta \quad \forall t \in\left(\rho_{n}, \rho_{n}^{*}\right), \\
& x_{i}(t) \geqslant \beta \quad \forall t \in\left(\rho_{n}^{*}, \rho_{n+1}\right) .
\end{aligned}
$$

For any $t \geqslant \rho_{1}$, if $t \in\left(\rho_{n}, \rho_{n}^{*}\right]$ for some integer $n$, we first of all show that $\rho_{n}$ must be an impulsive time. Otherwise, there exists a positive constant $\delta$ such that there is no pulse in the interval $\left(\rho_{n}, \rho_{n}+\delta\right) \subset\left(\rho_{n}, \rho_{n}^{*}\right]$. Then for any $t \in\left(\rho_{n}, \rho_{n}+\delta\right)$, from system (24) we have $x_{i}(t)>x_{i}\left(\rho_{n}^{+}\right)+\beta\left(t-\rho_{n}\right)>x_{i}\left(\rho_{n}\right) \geqslant$ $\beta$, which leads to a contradiction.

If there is only one impulsive time in the interval $\left(\rho_{n}, \rho_{n}^{*}\right]$ (which must be $\left.\rho_{n}\right)$, then from system (24) we get

$$
x_{i}(t)>x_{i}\left(\rho_{n}^{+}\right)+\beta\left(t-\rho_{n}\right) \geqslant \widetilde{h}_{i} x_{i}\left(\rho_{n}\right)+\beta\left(t-\rho_{n}\right)>\widetilde{h}_{i} \beta .
$$

If there are at least twice pulses in $\left(\rho_{n}, \rho_{n}^{*}\right]$, then we can denote $\left(\rho_{n}, \rho_{n}^{*}\right]=\bigcup_{p=p_{0}}^{q^{*}-1}\left(t_{p}, t_{p+1}\right] \bigcup\left(t_{q^{*}}, \rho_{n}^{*}\right]$, where $t_{p_{0}}=\rho_{n}$, $q^{*}>p_{0}, p_{0}, p$ and $q^{*}$ are some positive integers. Hence, for any $t \in\left(t_{p}, t_{p+1}\right]$, there is $x_{i}(t)>x_{i}\left(t_{p}^{+}\right)+\beta\left(t-t_{p}\right)$ from system (24). Moreover, if $p=p_{0}$, we have

$$
x_{i}(t)>x_{i}\left(t_{p}^{+}\right)+\beta\left(t-t_{p}\right) \geqslant \widetilde{h}_{i} x_{i}\left(\rho_{n}\right)+\beta\left(t-\rho_{n}\right)>\widetilde{h}_{i} \beta .
$$

If $p>p_{0}$, then

$$
\begin{aligned}
x_{i}(t)> & x_{i}\left(t_{p}^{+}\right)+\beta\left(t-t_{p}\right) \geqslant \widetilde{h}_{i}\left[x_{i}\left(t_{p-1}^{+}\right)+\beta\left(t_{p}-t_{p-1}\right)\right] \\
& +\beta\left(t-t_{p}\right)>\widetilde{h}_{i} \beta \tau_{0} .
\end{aligned}
$$

However, when $t \in\left(t_{q^{*}}, \rho_{n}^{*}\right]$, then

$$
\begin{aligned}
x_{i}(t)> & x_{i}\left(t_{q^{*}}^{+}\right)+\beta\left(t-t_{q^{*}}\right) \\
\geqslant & \widetilde{h}_{i}\left[x_{i}\left(t_{q^{*}-1}^{+}\right)+\beta\left(t_{q^{*}}-t_{q^{*}-1}\right)\right] \\
& +\beta\left(t-t_{q^{*}}\right)>\widetilde{h}_{i} \beta \tau_{0} .
\end{aligned}
$$

It follows from (28)-(31) that

$$
x_{i}(t) \geqslant \min \left\{\widetilde{h}_{i} \beta, \widetilde{h}_{i} \beta \tau_{0}\right\}
$$

for all $t \in\left(\rho_{n}, \rho_{n}^{*}\right]$ and any $i \in I$.

If there is an integer $n$ such that $t \in\left(\rho_{n}^{*}, \rho_{n+1}\right]$, obviously we have $x_{i}(t) \geqslant \beta$. Therefore, for Case 3 we always have

$$
x_{i}(t) \geqslant \min \left\{\widetilde{h}_{i} \beta, \widetilde{h}_{i} \beta \tau_{0}, \beta\right\}
$$

for all $t \geqslant \rho_{1}$ and any $i \in I$.

From (26) and (33), choose $m=\min _{i \in I}\left\{\widetilde{h}_{i} \beta, \widetilde{h}_{i} \beta \tau_{0}, \beta\right\} / 2$. Then we finally have that (12) holds. System (1) is permanent. The proof of Theorem 3 is completed.
Remark 4. It follows from Theorem 3 that system (1) is permanent if there is a positive average growth rate (which does not include the dispersal entrance) in one time period $T$ in any patch $i_{0}\left(i_{0} \in I\right)$. In paper [4], the authors showed an interesting result that the dispersal species without impulses is permanent in all other patches if it is permanent in a patch. However, we extend this result to a periodic case with impulses.

Remark 5. In paper [18], we studied an impulsive periodic predator-prey system with Holling type III functional response and diffusion, and the paper mainly considers the influences of Holling type functional response and impulses. The conditions of the main result Theorem 3 require the minimum of the coefficients. However, in this paper, we consider a single-species logistic system. Although the predator is not involved in the model, which is simple than the model in [18], a more accurate and reasonable condition is established in the present paper; that is, in dispersal system, species with impulses is permanent in all patches if it is permanent in a patch, which improves the minimum conditions in the previous paper.

Theorem 6. System (1) is extinct if conditions

$$
\int_{0}^{T} a(t) \mathrm{d} t>0, \quad \int_{0}^{T} \gamma(t) \mathrm{d} t+\sum_{k=1}^{q} \ln h_{k} \leqslant 0
$$

hold, where $\gamma(t)=\max _{i \in I}\left\{r_{i}(t)-\sum_{j=1}^{n} D_{i j}(t)+\sum_{j=1}^{n} D_{j i}(t)\right\}$ for all $t \in[0, T]$, and $a(t)$ and $h_{k}$ are defined in Theorem 1 .

Proof. In fact, from (34), for any constant $\varepsilon>0$, there is a positive constant $\widetilde{\delta}$ such that

$$
\int_{0}^{T}\left(\gamma(t)-\frac{\varepsilon a(t)}{n}\right) \mathrm{d} t+\sum_{k=1}^{q} \ln h_{k}<-\widetilde{\delta} .
$$

Define $V(t)=\sum_{i=1}^{n} x_{i}(t)$. When $t \neq t_{k}$, calculating the rightupper derivative of $V(t)$, we have

$$
\begin{aligned}
& D^{+} V(t) \\
& \begin{array}{l}
=\sum_{i=1}^{n} \dot{x}_{i}(t)=\sum_{i=1}^{n} x_{i}(t)\left(r_{i}(t)-\sum_{j=1}^{n} D_{i j}(t)\right. \\
\left.\quad+\sum_{j=1}^{n} D_{j i}(t)-a_{i}(t) x_{i}(t)\right) \\
\leqslant \gamma(t) V(t)-a(t) \sum_{i=1}^{n} x_{i}^{2}(t) \leqslant V(t)\left(\gamma(t)-\frac{a(t)}{n V(t)}\right) .
\end{array}
\end{aligned}
$$

When $t=t_{k}$, we obtain $V\left(t_{k}^{+}\right)=\sum_{i=1}^{n} x_{i}\left(t_{k}^{+}\right)=\sum_{i=1}^{n} h_{i k} x_{i}\left(t_{k}\right) \leqslant$ $\max _{i \in I}\left\{h_{i k}\right\} V\left(t_{k}\right)=h_{k} V\left(t_{k}\right)$. From this and (35), a similar argument as in the proof of (7), we can obtain $V(t) \leqslant$ $\varepsilon \exp (\gamma(t) T+H)$ for $t \geqslant 0$. Then from the arbitrariness of $\varepsilon$, we obtain $V(t) \rightarrow 0$ as $t \rightarrow \infty$. Finally, we have $x_{i}(t) \rightarrow 0$ as $t \rightarrow \infty$ for all $i \in I$. This completes the proof of Theorem 6 . 
Remark 7. It can be seen from Theorem 6 that system (1) is always extinct if, in one time period $T$, there are a positive density-dependent coefficient and a nonpositive average growth rate (which includes the dispersal entrance) in the time period in each patch $i(i \in I)$.

\section{Periodic Solutions}

In this section, by constructing an appropriate Lyapunov function, sufficient conditions for the existence of the unique globally attractively positive $T$-periodic solution of system (1) are established.

Let $x(t)=\left(x_{1}(t), x_{2}(t), \ldots, x_{n}(t)\right)$ and $x^{*}(t)=\left(x_{1}^{*}(t)\right.$, $\left.x_{2}^{*}(t), \ldots, x_{n}^{*}(t)\right)$ be any two positive solutions of system (1). From Theorem 3 , we can obtain that there are constants $A>0$ and $B>0$ such that

$$
A \leqslant x_{i}(t), \quad x_{i}^{*}(t) \leqslant B \quad \forall t \geqslant 0, i \in I .
$$

Theorem 8. Suppose all the conditions of Theorem 3 hold. Moreover, if

$$
\int_{0}^{T} \beta(t) \mathrm{d} t>0
$$

where $\beta(t)=\min _{i \in I}\left\{a_{i}(t)-\sum_{j=1}^{n} D_{j i}(t) / A\right\} \geqslant 0, t \in[0, T]$, then system (1) has a unique globally attractively positive $T$ periodic solution $x^{*}(t)=\left(x_{1}^{*}(t), x_{2}^{*}(t), \ldots, x_{n}^{*}(t)\right)$; that is, any positive solution $x(t)=\left(x_{1}(t), x_{2}(t), \ldots, x_{n}(t)\right)$ of system $(1)$ satisfies

$$
\lim _{t \rightarrow \infty}\left(x_{i}(t)-x_{i}^{*}(t)\right)=0, \quad i \in I \text {. }
$$

Proof. Choose Lyapunov function $V(t)=\sum_{i=1}^{n} \mid \ln x_{i}(t)-$ $\ln x_{i}^{*}(t) \mid$. Since for any impulsive time $t_{k}$ we have

$$
\begin{aligned}
V\left(t_{k}^{+}\right) & =\sum_{i=1}^{n}\left|\ln x_{i}\left(t_{k}^{+}\right)-\ln x_{i}^{*}\left(t_{k}^{+}\right)\right| \\
& =\sum_{i=1}^{n}\left|\ln h_{i k} x_{i}\left(t_{k}\right)-\ln h_{i k} x_{i}^{*}\left(t_{k}\right)\right|=V\left(t_{k}\right),
\end{aligned}
$$

then $V(t)$ is continuous for all $t \geqslant 0$. On the other hand, from (37) we can obtain that for any $t \in R_{+}$and $t \neq t_{k}$

$$
\begin{aligned}
\frac{1}{B}\left|x_{i}(t)-x_{i}^{*}(t)\right| & \leqslant\left|\ln x_{i}(t)-\ln x_{i}^{*}(t)\right| \\
& \leqslant \frac{1}{A}\left|x_{i}(t)-x_{i}^{*}(t)\right| .
\end{aligned}
$$

For any $t \in R_{+}$and $t \neq t_{k}$, calculating the derivative of $V(t)$, we obtain

$$
\begin{aligned}
D^{+} V(t) & =\sum_{i=1}^{n} \operatorname{sgn}\left(x_{i}(t)-x_{i}^{*}(t)\right)\left(\frac{\dot{x}_{i}(t)}{x_{i}(t)}-\frac{\dot{x}_{i}^{*}(t)}{x_{i}^{*}(t)}\right) \\
& \leqslant \sum_{i=1}^{n}\left(-a_{i}(t)\left|x_{i}(t)-x_{i}^{*}(t)\right|\right)+\sum_{i=1}^{n} \sum_{j=1}^{n} \bar{D}_{i j}(t),
\end{aligned}
$$

where

$$
\bar{D}_{i j}(t)= \begin{cases}D_{i j}(t)\left(\frac{x_{j}(t)}{x_{i}(t)}-\frac{x_{j}^{*}(t)}{x_{i}^{*}(t)}\right), & x_{i}(t)>x_{i}^{*}(t), \\ D_{i j}(t)\left(\frac{x_{j}^{*}(t)}{x_{i}^{*}(t)}-\frac{x_{j}(t)}{x_{i}(t)}\right), & x_{i}(t)<x_{i}^{*}(t) .\end{cases}
$$

For all $t \geqslant 0$, we estimate $\bar{D}_{i j}(t)$ under the following two cases.

(i) If $x_{i}(t) \geqslant x_{i}^{*}(t)$, then $\bar{D}_{i j}(t) \leqslant D_{i j}(t) / x_{i}(t)\left(x_{j}(t)-\right.$ $\left.x_{j}^{*}(t)\right) \leqslant D_{i j}(t) / A\left|x_{j}(t)-x_{j}^{*}(t)\right|$.

(ii) If $x_{i}(t)<y_{i}(t)$, then $\bar{D}_{i j}(t) \leqslant D_{i j}(t) / x_{i}^{*}(t)\left(x_{j}(t)-\right.$ $\left.x_{j}^{*}(t)\right) \leqslant D_{i j}(t) / A\left|x_{j}(t)-x_{j}^{*}(t)\right|$.

It follows from the estimation of $\bar{D}_{i j}(t)$ and (41) that

$$
\begin{aligned}
D^{+} V(t) \leqslant & \sum_{i=1}^{n}\left(-a_{i}(t)\left|x_{i}(t)-x_{i}^{*}(t)\right|\right) \\
& +\sum_{i=1}^{n} \sum_{j=1}^{n} \frac{D_{i j}(t)}{A\left|x_{j}(t)-x_{j}^{*}(t)\right|} \\
\leqslant & -\sum_{i=1}^{n}\left(a_{i}(t)-\sum_{j=1}^{n} \frac{D_{j i}(t)}{A}\right)\left|x_{i}(t)-x_{i}^{*}(t)\right| \\
\leqslant & -\beta(t) A V(t) .
\end{aligned}
$$

From this and condition (38), we have $V(t) \leqslant$ $V(0) \exp \left(-A \int_{0}^{t} \beta(s) \mathrm{d} s\right) \rightarrow 0$ as $t \rightarrow \infty$. Further more, from (41) we have that (39) holds.

Now let us consider the sequence $\left(x_{1}^{*}\left(m T, z_{0}\right), x_{2}^{*}(m T\right.$, $\left.\left.z_{0}\right), \ldots, x_{n}^{*}\left(m T, z_{0}\right)\right)=z\left(m T, z_{0}\right)$, where $m=1,2, \ldots$ and $z_{0}=\left(x_{1}^{*}(0), x_{2}^{*}(0), \ldots, x_{n}^{*}(0)\right)$. It is compact in the domain $[A, B]^{n}$ since $A \leqslant x_{i}^{*}(t) \leqslant B$ for all $t \geqslant 0$ and $i=$ $1,2, \ldots, n$. Let $\bar{z}$ be a limit point of this sequence, with $\bar{z}=\lim _{n \rightarrow \infty} z\left(m_{n} T, z_{0}\right)$. Then $z(T, \bar{z})=\bar{z}$. Indeed, since $z\left(T, z\left(m_{n} T, z_{0}\right)\right)=z\left(m_{n} T, z\left(T, z_{0}\right)\right)$ and $z\left(m_{n} T, z\left(T, z_{0}\right)\right)-$ $z\left(m_{n} T, z_{0}\right) \rightarrow 0$ as $m_{n} \rightarrow \infty$, we get

$$
\begin{aligned}
&\|z(T, \bar{z})-\bar{z}\|_{[A, B]^{n}} \\
& \leqslant\left\|z(T, \bar{z})-z\left(T, z\left(m_{n} T, z_{0}\right)\right)\right\|_{[A, B]^{n}} \\
&+\left\|z\left(T, z\left(m_{n} T, z_{0}\right)\right)-z\left(m_{n} T, z_{0}\right)\right\|_{[A, B]^{n}} \\
& \quad+\left\|z\left(m_{n} T, z_{0}\right)-\bar{z}\right\|_{[A, B]^{n}} \longrightarrow 0, \quad n \longrightarrow \infty .
\end{aligned}
$$

The sequence $z\left(m T, z_{0}\right), m=1,2, \ldots$ has a unique limit point. On the contrary, let the sequence have two limit points $\bar{z}=\lim _{n \rightarrow \infty} z\left(m_{n} T, z_{0}\right)$ and $\widetilde{z}=\lim _{n \rightarrow \infty} z\left(m_{n} T, z_{0}\right)$. Then, taking into account (39) and $\widetilde{z}=z\left(m_{n} T, \widetilde{z}\right)$, we have

$$
\begin{aligned}
&\|\bar{z}-\widetilde{z}\|_{[A, B]^{n}} \\
& \quad \leqslant\left\|\bar{z}-z\left(m_{n} T, z_{0}\right)\right\|_{[A, B]^{n}} \\
& \quad+\left\|z\left(m_{n} T, z_{0}\right)-\widetilde{z}\right\|_{[A, B]^{n}} \longrightarrow 0, \quad n \longrightarrow \infty,
\end{aligned}
$$


and hence $\bar{z}=\widetilde{z}$. The solution $\left(x_{1}^{*}(t, \bar{z}), x_{2}^{*}(t, \bar{z}), \ldots, x_{n}^{*}(t, \bar{z})\right)$ is the unique periodic solution of system (1). By (39), it is globally attractive. This completes the proof of Theorem 8 .

\section{Numerical Simulation and Discussion}

In this paper, we have investigated a class of single-species periodic logistic system with impulses and dispersal in $n$ different patches. By means of inequality estimation technique and Lyapunov function, we gave the criteria for the permanence, extinction, and existence of a unique globally stable positive periodic solution of system (1).

In order to testify the validity of our results and present a more in-depth problem for further discussion, we discuss the following two patches $T$-periodic dispersal system:

$$
\begin{gathered}
\dot{x}_{1}(t)=x_{1}(t)\left(r_{1}(t)-a_{1}(t) x_{1}(t)\right) \\
\quad+D_{12}(t)\left(x_{2}(t)-x_{1}(t)\right), \\
\dot{x}_{2}(t)=x_{2}(t)\left(r_{2}(t)-a_{2}(t) x_{2}(t)\right) \\
+D_{21}(t)\left(x_{1}(t)-x_{2}(t)\right), \\
t \neq t_{k}, \\
x_{1}\left(t_{k}^{+}\right)=h_{1 k} x_{1}\left(t_{k}\right), \\
x_{2}\left(t_{k}^{+}\right)=h_{2 k} x_{2}\left(t_{k}\right), \\
k=1,2, \ldots
\end{gathered}
$$

We take $r_{1}(t)=5-|\sin (\pi / 2) t|, r_{2}(t)=2.5+0.5 \cos \pi t$, $a_{1}(t)=1.5, a_{2}(t)=0.8, D_{12}(t)=1.2, D_{21}(t)=0.7, h_{1 k}=$ $1.2, h_{2 k}=0.8$, and $t_{k}=0.1 k, k=1,2, \ldots$. Obviously, $r(t)=$ $\max \left\{r_{1}(t), r_{2}(t)\right\}=5-|\sin (\pi / 2) t|, a(t)=\min \left\{a_{1}(t), a_{2}(t)\right\}=$ $0.8, h_{k}=\max \left\{h_{1 k}, h_{2 k}\right\}=1.2$, and system (47) is periodic with period $T=2$. For $q=20$, we have $t_{k+q}=t_{k}+T, h_{1(k+q)}=h_{1 k}$, and $h_{2(k+q)}=h_{2 k}$ for all $k=1,2, \ldots$. It is easy to verify that $h_{k}(t, \mu)$ and $h_{i k}(t, \mu)(i=1,2)$ are bounded for all $t \in R_{+}$and $\mu \in[0, T]$. Further more, since

$$
\begin{gathered}
\int_{0}^{T} a(t) \mathrm{d} t=1.6>0, \\
\int_{0}^{T}\left(r_{1}(t)-D_{12}(t)\right) \mathrm{d} t+\sum_{k=1}^{q} \ln h_{1 k} \\
=9.9732>0 \quad\left(i_{0}=1\right),
\end{gathered}
$$

all the conditions of Theorem 3 are satisfied. Hence, system (47) is permanent. See Figures 1 and 2.

However, if the survival environment of the two patches is austere, the intrinsic growth rates will be negative. Hence, if we take $r_{1}(t)=-1-|\sin (\pi / 2) t|, r_{2}(t)=-4+0.5 \cos \pi t$ and all other parameters are retained, then we obtain $\gamma(t)=$ $\max \left\{r_{1}(t)-D_{12}(t)+D_{21}(t), r_{2}(t)-D_{21}(t)+D_{12}(t)\right\}=-1.5-$ $|\sin (\pi / 2) t|$ and

$$
\int_{0}^{T} \gamma(t) \mathrm{d} t+\sum_{k=1}^{q} \ln h_{k}=-0.6268<0
$$

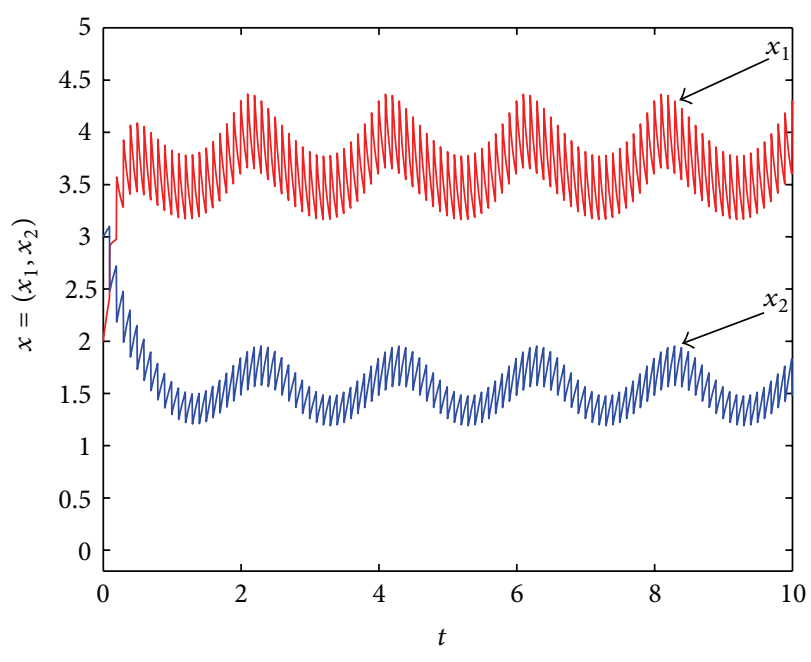

FIGURE 1: The time series of the permanence of species $x$.

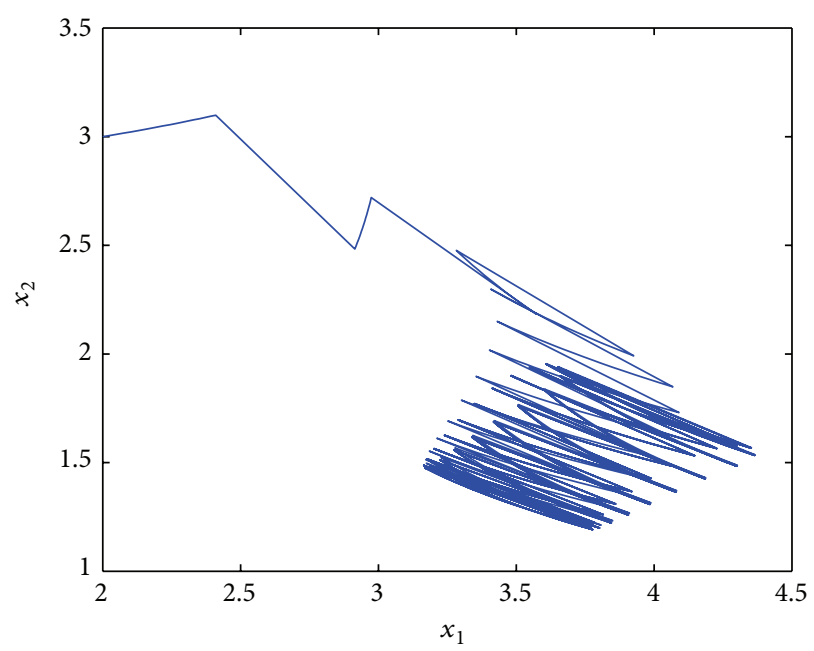

FIgURE 2: The phase of the permanence of species $x$.

hence conditions of Theorem 6 are satisfied. From Theorem 6 we find that any positive solution of system (47) will be extinct. See Figure 3.

From the illustrations of the theorems, we note that there is a great difference on the choice of the intrinsic growth rates $r_{i}(t)(i=1,2)$, which guarantee that the system is permanent or extinct. These differences make us want to know what results will be if all the parameters satisfy

$$
\begin{gathered}
\int_{0}^{T}\left(r_{i}(t)-\sum_{j=1}^{2} D_{i j}(t)\right) \mathrm{d} t+\sum_{k=1}^{q} \ln h_{i k} \leqslant 0 \quad i=1,2, \\
\int_{0}^{T} \gamma(t) \mathrm{d} t+\sum_{k=1}^{q} \ln h_{k}>0 .
\end{gathered}
$$



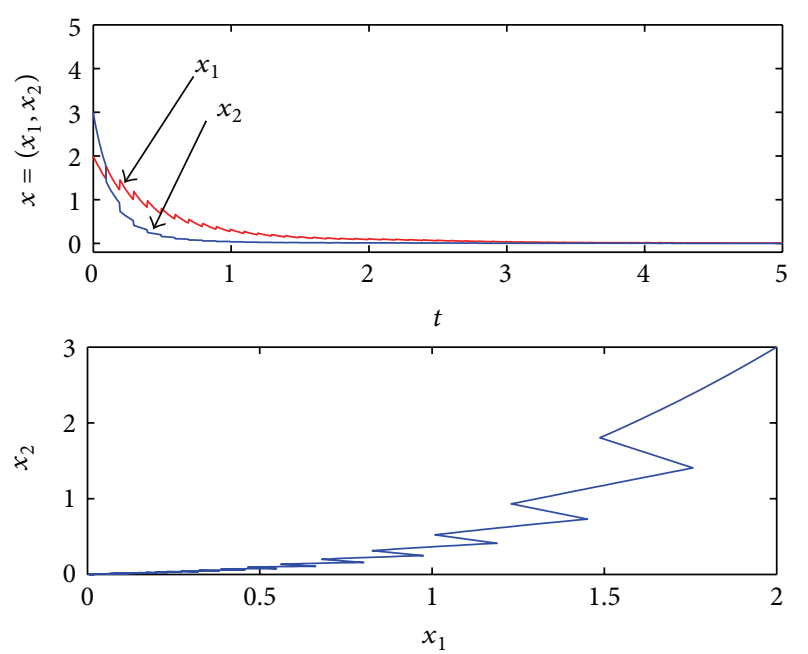

FIGURE 3: The time series and phase of the extinction of species $x$.

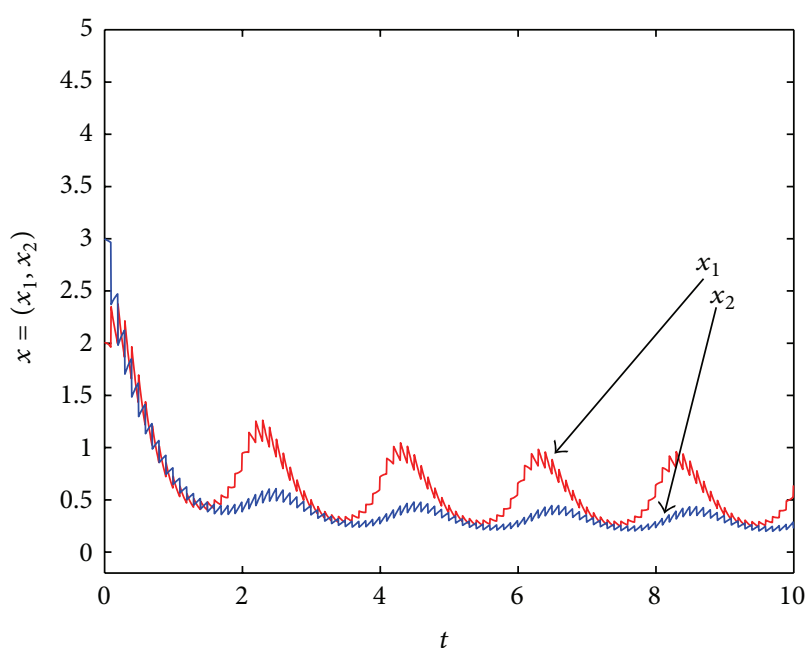

FIGURE 4: The time series of the permanence of species $x$.

For this aim, we choose $r_{1}(t)=2.55-5|\sin (\pi / 2) t|, r_{2}(t)=$ $2+0.5 \cos \pi t$ and all other parameters are retained; then

$$
\begin{gathered}
\int_{0}^{T}\left(r_{1}(t)-D_{12}(t)\right) \mathrm{d} t+\sum_{k=1}^{q} \ln h_{1 k}=-0.0198<0, \\
\int_{0}^{T}\left(r_{2}(t)-D_{21}(t)\right) \mathrm{d} t+\sum_{k=1}^{q} \ln h_{2 k}=-1.8629<0, \\
\int_{0}^{T} \gamma(t) \mathrm{d} t+\sum_{k=1}^{q} \ln h_{k}=11.3732>0,
\end{gathered}
$$

and all the conditions of Theorems 3 and 6 are not satisfied. But from Figures 4 and 5 we find that the system is permanent.

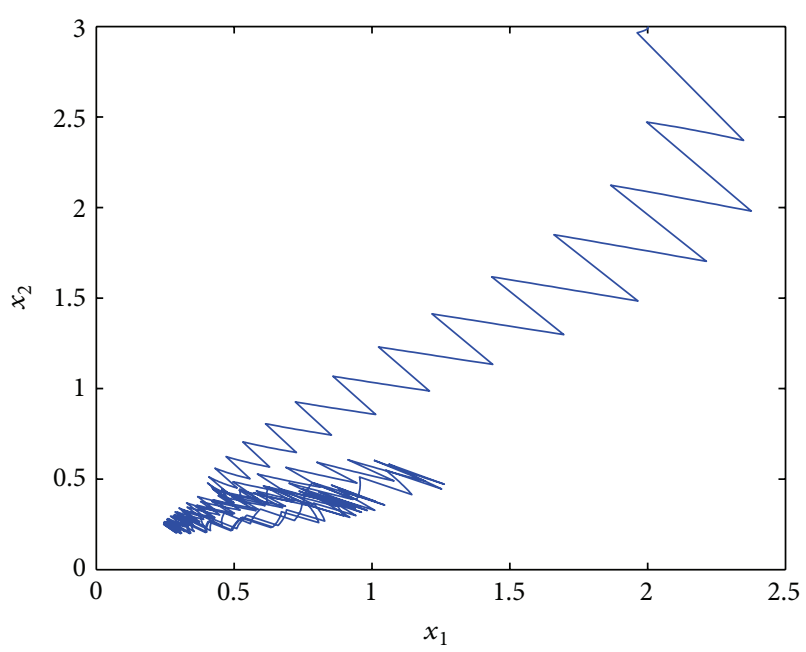

FIGURE 5: The phase of the permanence of species $x$.
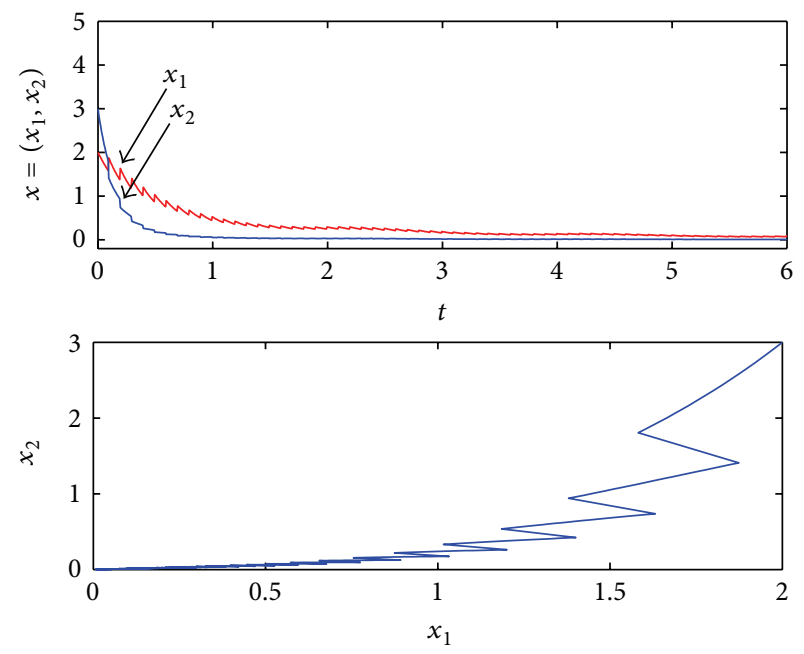

Figure 6: The time series and phase of the extinction of species $x$.

Furthermore, if we choose $r_{1}(t)=-0.5-|\sin (\pi / 2) t|$, $r_{2}(t)=-4+0.5 \cos \pi t$ and keep all other parameters, then we have

$$
\begin{gathered}
\int_{0}^{T}\left(r_{1}(t)-D_{12}(t)\right) \mathrm{d} t+\sum_{k=1}^{q} \ln h_{1 k}=-1.0268<0, \\
\int_{0}^{T}\left(r_{2}(t)-D_{21}(t)\right) \mathrm{d} t+\sum_{k=1}^{q} \ln h_{2 k}=-12.4629<0, \\
\int_{0}^{T} \gamma(t) \mathrm{d} t+\sum_{k=1}^{q} \ln h_{k}=1.3732>0,
\end{gathered}
$$

which do not satisfy conditions of any theorem. But, from Figure 6 we see that any positive solution of system (1) is extinct.

Remark 9. Through the above analysis, we realize that there is a little flaw of the finding conditions of the theorems. 
A challenging problem is to find some sufficient and necessary conditions (if the conditions hold, then the system will be permanent, otherwise, it will be extinct) to guarantee the permanence and extinction of the system.

Throughout Figures 1-6, we always take the initial condition $x(0)=\left(x_{1}(0), x_{2}(0)\right)=(2,3)$.

\section{Acknowledgments}

This work was supported in part by the National Natural Science Foundation of China (60702071), Program for New Century Excellent Talents in University (NCET06-0811), 973 National Basic Research Program of China (2010CB732501), Foundation of Sichuan Excellent Young Talents (09ZQ026-035), and Open Project of State Key Laboratory for Novel Software Technology of Nanjing University and Zhejiang Provincial Natural Science Foundation of China (Q13A010080).

\section{References}

[1] E. Beretta and Y. Takeuchi, "Global stability of single-species diffusion Volterra models with continuous time delays," Bulletin of Mathematical Biology, vol. 49, no. 4, pp. 431-448, 1987.

[2] H. I. Freedman and Y. Takeuchi, "Global stability and predator dynamics in a model of prey dispersal in a patchy environment," Nonlinear Analysis. Theory, Methods \& Applications, vol. 13, no. 8, pp. 993-1002, 1989.

[3] Y. Kuang and Y. Takeuchi, "Predator-prey dynamics in models of prey dispersal in two-patch environments," Mathematical Biosciences, vol. 120, no. 1, pp. 77-98, 1994.

[4] Z. Teng and Z. Lu, "The effect of dispersal on single-species nonautonomous dispersal models with delays," Journal of Mathematical Biology, vol. 42, no. 5, pp. 439-454, 2001.

[5] J. Zhang, L. Chen, and X. D. Chen, "Persistence and global stability for two-species nonautonomous competition LotkaVolterra patch-system with time delay," Nonlinear Analysis. Theory, Methods \& Applications, vol. 37, no. 8, pp. 1019-1028, 1999.

[6] R. Mchich, P. Auger, and J.-C. Poggiale, "Effect of predator density dependent dispersal of prey on stability of a predatorprey system," Mathematical Biosciences, vol. 206, no. 2, pp. 343356, 2007.

[7] Y. Takeuchi, J. Cui, R. Miyazaki, and Y. Saito, "Permanence of delayed population model with dispersal loss," Mathematical Biosciences, vol. 201, no. 1-2, pp. 143-156, 2006.

[8] L. Zhang and Z. Teng, "Permanence for a class of periodic timedependent competitive system with delays and dispersal in a patchy-environment," Applied Mathematics and Computation, vol. 188, no. 1, pp. 855-864, 2007.

[9] Z. Teng and L. Chen, "Permanence and extinction of periodic predator-prey systems in a patchy environment with delay," Nonlinear Analysis. Real World Applications, vol. 4, no. 2, pp. 335-364, 2003.

[10] V. Lakshmikantham, D. D. Bainnov, and P. S. Simeonov, Theory of Impulsive Differential Equations, vol. 6, World Scientific Press, Singapore, 1989.

[11] S. Ahmad and I. M. Stamova, "Asymptotic stability of an $N$ dimensional impulsive competitive system," Nonlinear Analysis. Real World Applications, vol. 8, no. 2, pp. 654-663, 2007.
[12] M. U. Akhmet, M. Beklioglu, T. Ergenc, and V. I. Tkachenko, "An impulsive ratio-dependent predator-prey system with diffusion," Nonlinear Analysis. Real World Applications, vol. 7, no. 5, pp. 1255-1267, 2006.

[13] X.-Z. Meng, L.-S. Chen, and Q.-X. Li, "The dynamics of an impulsive delay predator-prey model with variable coefficients," Applied Mathematics and Computation, vol. 198, no. 1, pp. 361374, 2008.

[14] S. H. Saker and J. O. Alzabut, "Existence of periodic solutions, global attractivity and oscillation of impulsive delay population model," Nonlinear Analysis. Real World Applications, vol. 8, no. 4, pp. 1029-1039, 2007.

[15] J. Hou, Z. Teng, and S. Gao, "Permanence and global stability for nonautonomous $N$-species Lotka-Valterra competitive system with impulses," Nonlinear Analysis. Real World Applications, vol. 11, no. 3, pp. 1882-1896, 2010.

[16] X. Liu and L. Chen, "Global dynamics of the periodic logistic system with periodic impulsive perturbations," Journal of Mathematical Analysis and Applications, vol. 289, no. 1, pp. 279-291, 2004.

[17] X. Wang, W. Wang, and X. Lin, "Dynamics of a periodic Watt-type predator-prey system with impulsive effect," Chaos, Solitons and Fractals, vol. 39, no. 3, pp. 1270-1282, 2009.

[18] Z. Liu and S. Zhong, "An impulsive periodic predator-prey system with Holling type III functional response and diffusion," Applied Mathematical Modelling, vol. 36, no. 12, pp. 5976-5990, 2012. 


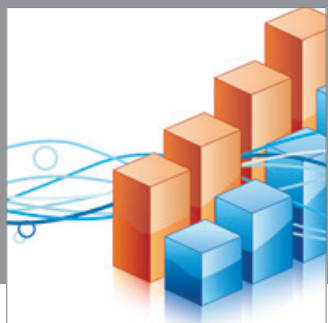

Advances in

Operations Research

mansans

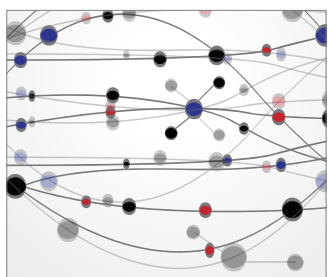

The Scientific World Journal
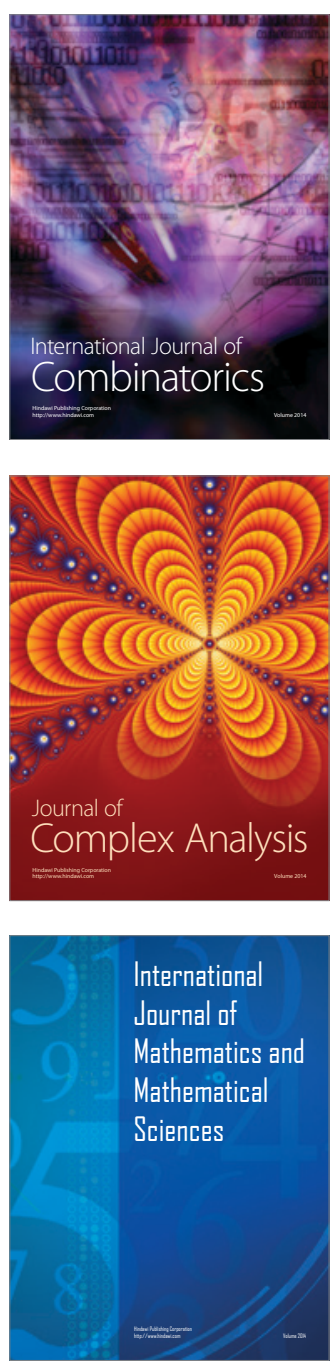
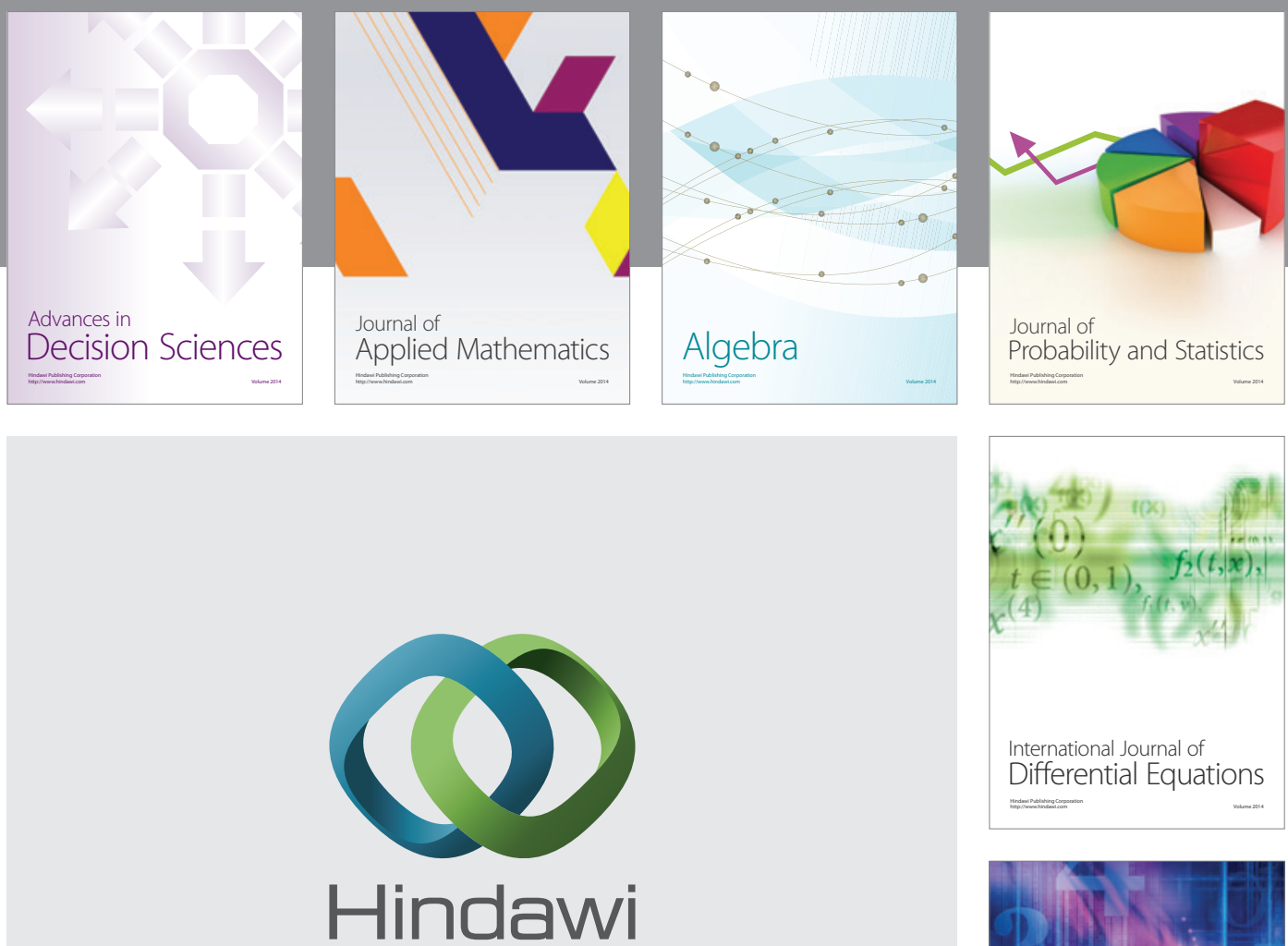

Submit your manuscripts at http://www.hindawi.com
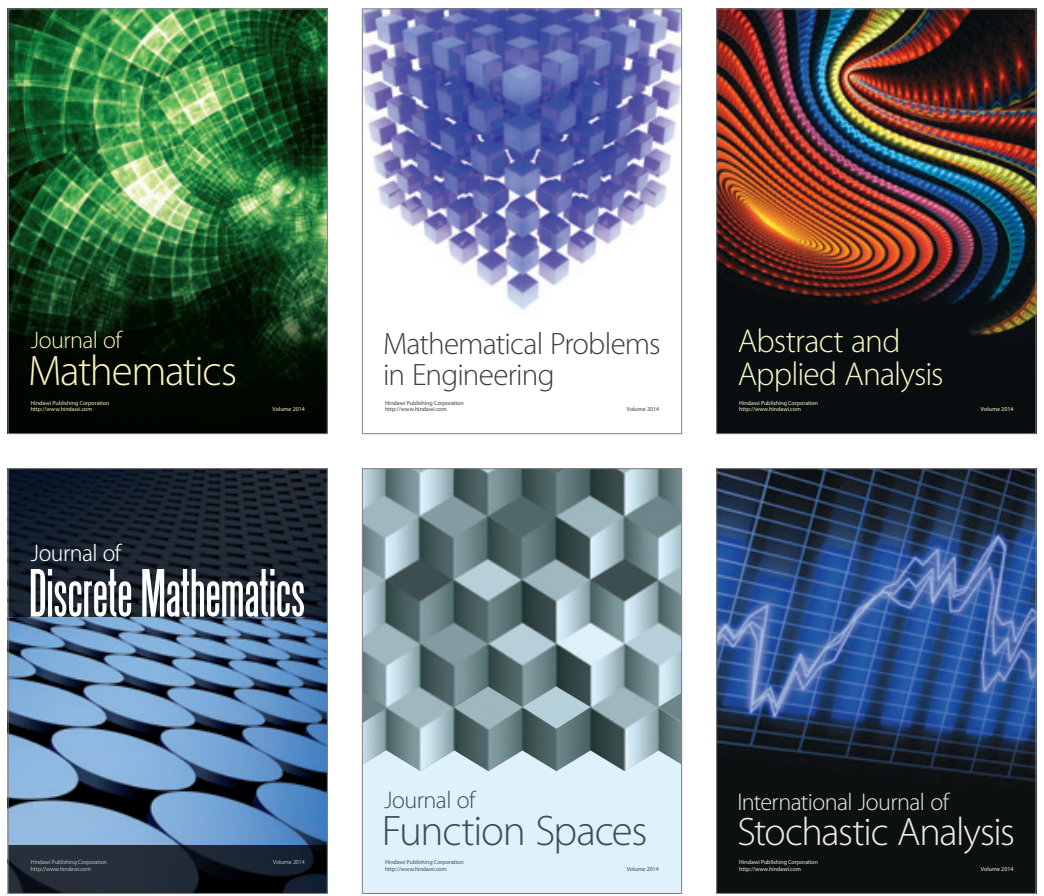

Journal of

Function Spaces

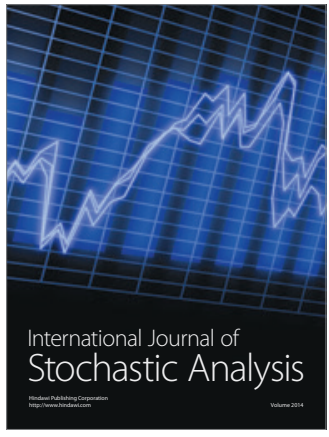

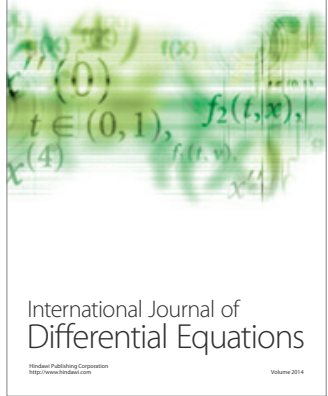
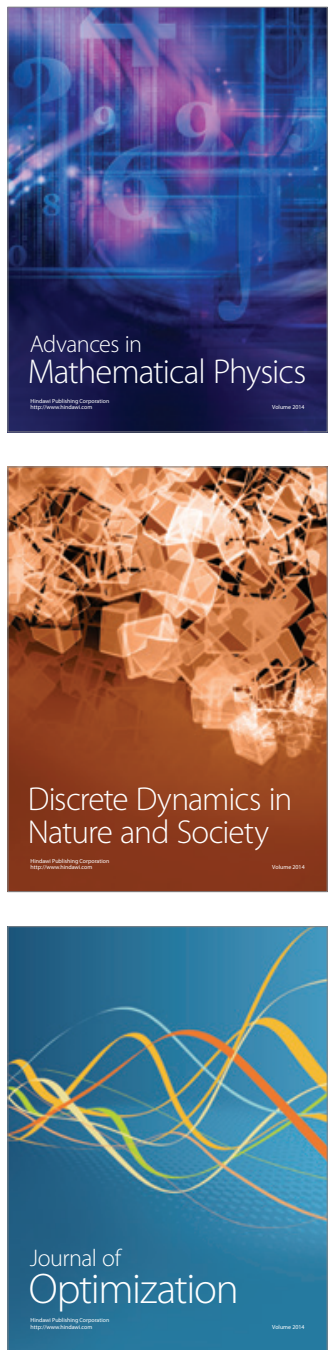